\title{
LOS CONJUNTOS FAUNÍSTICOS PLEISTOCÉNICOS DE CUEVA LAS GUANACAS (REGIÓN DE AISEN, PATAGONIA CHILENA): ALCANCES TAXONÓMICOS Y TAFONÓMICOS
}

\author{
RAFAEL LABARCA E., FELIPE FUENTES M."' Y FRANCISCO MENA L."'
}

\begin{abstract}
RESUMEN
Este trabajo entrega los resultados de los análisis taxonómicos y tafonómicos efectuados a los conjuntos faunísticos de las capas más profundas del sitio cueva Las Guanacas (Región de Aisén). Se constata la presencia de ocho taxones mayores, cuatro de ellos extintos (Mylodon darwini Owen, Macrauchenia patachonica Owen, Hippidion saldiasi Roth y Camelidae indet.) y cuatro que aún habitan el sector o sus alrededores (Hippocamelus bisulcus Molina, Pudu pudu Molina, Lama guanicoe Müller y Dusicyon culpaeus Molina). La evidencia tafonómica descarta una ocupación cultural temprana, apuntando en cambio a una utilización natural de la cueva por una o más especies adicionales, reconocibles a partir de distintas líneas de evidencia (etología de los predadores finipleistocénicos, taxones presentes, frecuencia de partes esqueletarias, modificaciones naturales). Se plantea que el principal agente responsable de la formación del conjunto faunístico de Las Guanacas habría sido un carnívoro (al menos un felino de tamaño medio, posiblemente Panthera onca Linnaeus), el que habría implementado distintas estrategias de obtención de las carcasas (caza y carroñeo) hacia los 13.200 AP.
\end{abstract}

PALABRAS CLAVE: Patagonia central, Pleistoceno final, taxonomía, tafonomía, acción de carnívoros.

\section{THE PLEISTOCENE ARCHAEOFAUNA OF CUEVA LAS GUANACAS (REGIÓN DE AISEN, CHILEAN PATAGONIA): TAXONOMIC AND TAPHONOMIC IMPLICATIONS}

\section{ABSTRACT}

The results of taxonomic and taphonomic analyses from the lowermost layers of the Cueva Las Guanacas archaeological site (XI Region, Chilean Central Patagonia) are presented. Eight major taxa have been identified, among them four extinct (Mylodon darwini Owen, Macrauchenia patachonica Owen, Hippidion saldiasi Roth and Camelidae indet.) and other four currently living on the valley or nearby areas (Hippocamelus bisulcus Molina, Pudu pudu Molina, Lama guanicoe Müller y Dusicyon culpaeus Molina). The taphonomic analysis confirms the non-cultural nature of the early levels and instead backs

\footnotetext{
* Programa de doctorado de la Universidad del Centro de la Provincia de Buenos Aires (Argentina) r.labarca.e@gmail.com

* Universidad Bolivariana. Huérfanos 2970, Sede Santiago, Chile. felipe.fuentes.mucherl@gmail.com

$\because \quad$ Museo Chileno de Arte Precolombino. Bandera 361, Santiago, Chile. fmena@museoprecolombino.cl
} 
up the idea of non human predators occupying the cave. Resorting to many different lines of evidence (ethology of Pleistocene predators, observed taxonomic and skeletal profiles, gastric acid corrosion and other damages), it is claimed that the agent responsible for the Pleistocene/Holocene bone assemblage at cueva Las Guanacas were carnivores (including a mid-sized feline, possibly Panthera onca Linnaeus). These predators would have had deveoped several strategies to obtain the carcasses, including active hunt and scavenging around 13.200 BP.

KEY WORDS: Central Patagonia, final Pleistocene, taxonomy, taphonomy, carnivores' action.

\section{INTRODUCCIÓN}

Desde su primera publicación (Mena 1983) ha estado en duda la naturaleza antrópica de los niveles basales de cueva Las Guanacas. Las evidencias de una ocupación Holoceno temprana en sectores esteparios de Baño Nuevo-1 (Mena y Stafford 2006) y El Chueco (Reyes et al. 2007) y-por el contrario-la porfiada ausencia de estos materiales en la inmediata vecindad hacia el sur (Mengoni et al. 2008), hacen especialmente importante evaluar toda evidencia disponible y no partir de supuestos fáciles o hipótesis generales para determinar la primera ocupación humana en estos ambientes, que por lo demás corresponden a la transición hacia el bosque montano. Ello nos ha llevado a reevaluar minuciosamente los materiales faunísticos recuperados bajo la primera ocupación humana fechada en el sitio (5.340 \pm 180 AP). Adelantándonos a los resultados, la revisión de las modificaciones observables en los restos óseos prácticamente descartó una temprana ocupación en el lugar, por lo que, en principio, la tarea ya estaba realizada Se trataba pues, de un contexto paleontológico, por lo que, en teoría, se alejaba de la incumbencia y del interés de los arqueólogos. Sin embargo, el análisis de un conjunto óseo temprano sin asociación cultural desde una perspectiva zooarqueológica y particularmente tafonómica, además de contribuir a un mejor conocimiento y comprensión de las condiciones paleoecológicas en el valle del río Ibáñez en la transición Pleistoceno-Holoceno, permite generar una "línea de base" o "telón de referencia" para entender ocupaciones humanas de esta época (en caso de que eventualmente se hallen a futuro en similares ambientes o en el mismo valle) o -por el contrario- para interpretar su inexistencia. Mal que mal, la coincidencia de la primera presencia humana en América con el cambio ambiental representado por la transición al Holoceno y la extinción de varias especies animales, está abierta a interpretaciones alternativas, incluyendo la imposibilidad de la presencia humana en coexistencia con grandes predadores, ya que debieron competir por las mismas presas y espacios (p.e cuevas, Graham y Lundelius 1984). Estos componentes finipleistocénicos, por tanto, que debido a su elevada fragmentación y escasa diagnosis, pueden ser de poco interés para la mayoría de los paleontólogos, son de gran utilidad en la perspectiva de la arqueología, aunque no estén directamente asociados a restos culturales. Es por ello que están siendo cada vez más estudiados por los zooarqueólogos, aunque lamentablemente esta tendencia está aun en gran parte confinada a Patagonia (p.e. Borrero et al. 1997; Martin et al. 2004).

\section{ANTECEDENTES}

Cueva Las Guanacas (RI-16) es fruto del derrumbe de grandes bloques en la ladera noreste del cerro Nudo, sobre el salto del Ibáñez (46¹5' S 715'ㄴ) (Fig. 1). Conocida por los pobladores de la zona desde hace varios años dada su destacada presencia y la existencia en ella de pinturas rupestres, fue descrita sistemáticamente y publicada por primera vez por Felipe Bate (1970; Sade 2008: 41). Se encuentra ubicada a $350 \mathrm{mts}$ sobre el nivel del mar y aproximadamente $200 \mathrm{mts}$ sobre el punto de confluencia del río Claro en el río Ibáñez, en la parte superior de un faldeo empinado, unos 100 mts por debajo de un plano amesetado boscoso. El sitio corresponde a una cueva de $18 \mathrm{mts}$ de profundidad y 6 mts de ancho promedio (Fig. 2) en una zona de transición entre el piso estepario del valle y esta meseta. Mientras el primer ambiente está hoy dominado por pastos y arbustos xerófitos, siendo espacio privilegiado del guanaco y el ñandú (animales que hoy no se encuentran en el valle, pero de los que hay evidencia arqueológica), el ambiente 


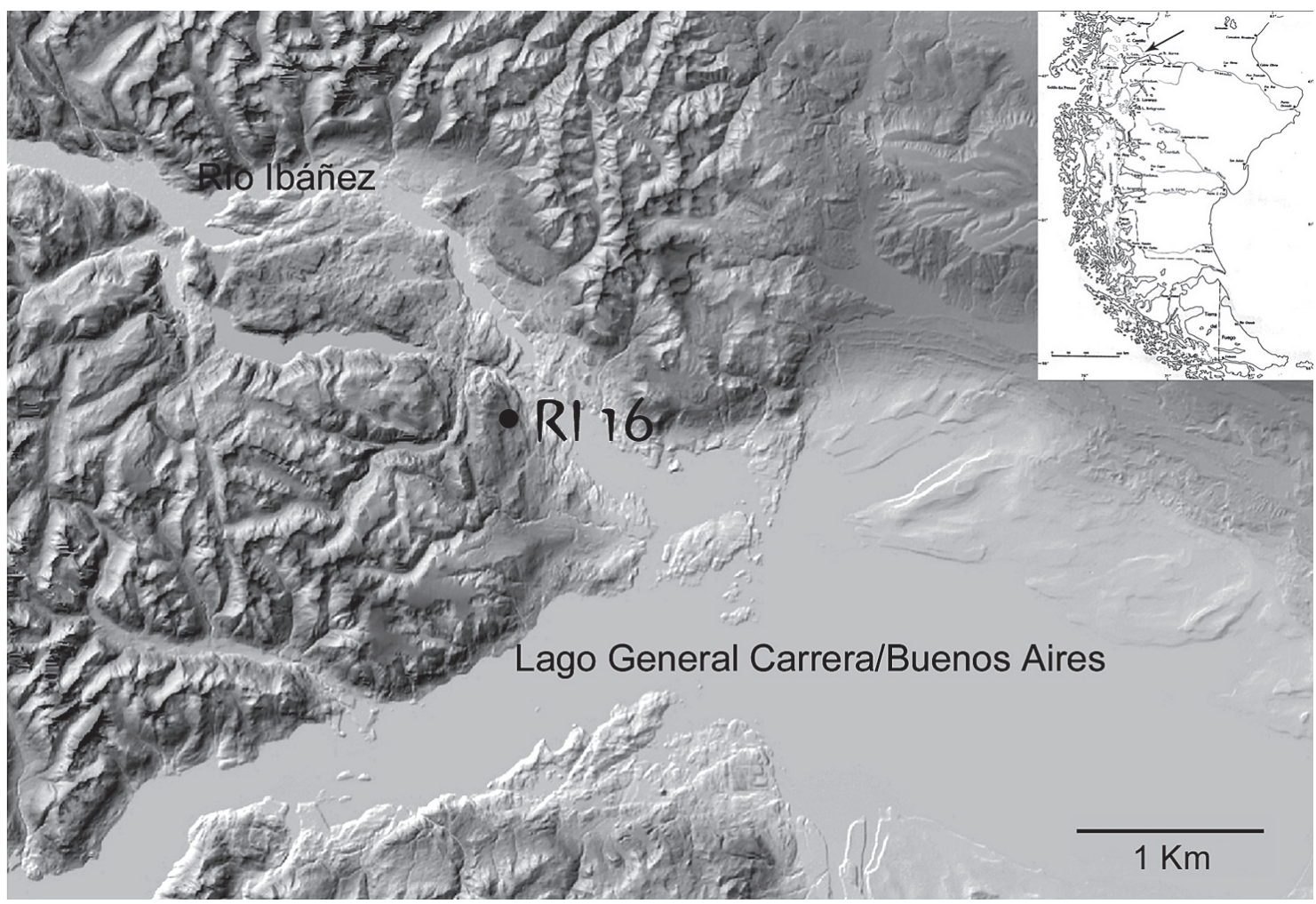

Fig. 1. Ubicación de cueva Las Guanacas.

boscoso alto debió sustentar a diversas aves de parque y lagunas, además de pudú y huemul (Fuentes y Mena 2008).

Excavado en 1982, 1983 (Mena 1983) y 1997 (Mena 1997), el sitio revela una clara estratigrafía, debido en gran parte a la depositación de "cenizas" volcánicas de sucesivas erupciones del volcán Hudson (Naranjo y Stern 1998). Tanto la orientación de la boca de la cueva, como la inclinación natural hacia adentro (a diferencia de la mayoría de las cuevas estructurales, cuyo talud cae hacia fuera) han condicionado una sedimentación rápida y bastante regular, sobre todo hacia el fondo. El relleno $y$ posterior pisoteo de ovejas define una tendencia a la horizontalidad, de modo que los "estratos" aparecen más delgados y mezclados hacia el frente generando incertidumbres cronológicas que -como veremos- nos llevaron a finalmente decidir excluir de este análisis los restos recuperados allí.

En todo caso, creemos que es proyectable a todo el sitio la secuencia estratigráfica claramente reconocida en las cuadrículas estudiadas. Esta comienza a más de tres metros bajo el suelo actual con un potente estrato de derrumbe (base indefinida; espesor superior a $1,5 \mathrm{~m}$ ) conformado por bloques de hasta 2 mts de diámetro que deben haber definido una especie de "cama" sobre la que viajaron los enormes bloques que conformaron la cueva (Capa 12) (Fig. 2). Esta es muy similar a las Capas 10 y 8, separadas sólo por lentes de arena volcánica (Capa 11; limo 20 cm de espesor y Capa 9; arena $10 \mathrm{~cm}$ espesor) (Fig. 2). En un primer momento se pensó que la Capa 10 contenía las evidencias de ocupación humana más tempranas en el sitio (lo que quedó descartado con las excavaciones de 1997 y el presente análisis). Un molar de équido extinto contenido en ella ha dado una fecha de $13275 \pm 30$ AP (UCIAMS- 53544) (AMS; 14.155 - $13.457 \mathrm{cal}$ AP 2 sigmas).

La Capa 7 corresponde a otra tephra volcánica, atribuible a una gran erupción del volcán Hudson hacia el 6.700 AP (Fig. 2).

La presencia humana sólo se detecta fehacientemente en el sitio en la superpuesta Capa 6 (Fig. 2), fechada en 5.340 $\pm 190 \mathrm{AP}$ (Beta-7635). Originalmente esta ocupación fue denominada "nivel cultural II", 


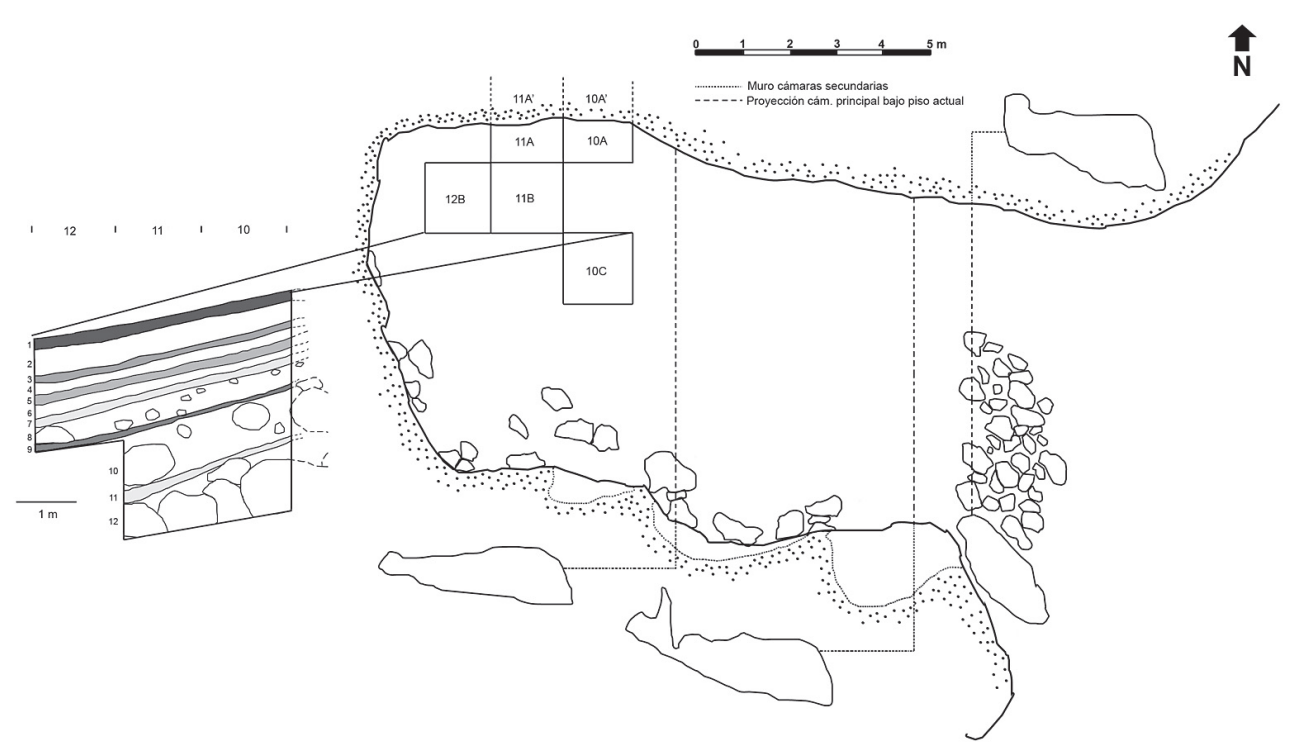

Fig. 2. Planta de la cueva Las Guanacas y corte estratigráfico de las unidades analizadas en el presente trabajo.

pero es de hecho el primer evento de ocupación humana registrado en el sitio, por lo que conviene referirse a él como "componente inferior".

Separado de éste por dos capas estériles (Capa 5 tephra $10 \mathrm{~cm}$ esp.; y Capa 4 pedregullo 5 $\mathrm{cm}$ esp.) hallamos las Capas 2 y 3, que representan un "componente superior" (antes "nivel cultural III"), fechado hacia el 500 AP (450 \pm 70 AP; Beta-7087en Capa 2) (Fig. 2).

Para nuestros fines, basta señalar que la secuencia se encuentra marcada por un potente estrato precultural y al menos dos eventos antrópicos discretos, separados nítidamente por caída de cenizas y derrumbes, cuyas capas fueron excluidas de este análisis.

\section{MATERIAL Y MÉTODOS}

Se trabajó con una muestra de 1585 especimenes óseos provenientes de las capas 9, 10, 11 y 12 de las cuadrículas 10A, 10A', 10C, 11A, 11A', 11B y $12 B$ de cueva Las Guanacas (Fig. 2) recuperados en todas las campañas de excavación. Se consideraron sólo las cuadrículas ubicadas al fondo de la cueva debido a que presentaban una mejor integridad estratigráfica, además de que concentraron el grueso de los restos de mamíferos extintos (Labarca, datos sin publicar). La identificación anatómica y taxonómica se logró a través de tres métodos complementarios, dependiendo de la naturaleza de las piezas revisadas. De esta forma, se utilizaron guías de osteología de referencia (Alberdi y Prado 2004; Altamirano 1983; Benavente et al. 1992; Pacheco et al. 1982), se realizaron comparaciones directas con esqueletos actuales y fósiles depositados en distintas instituciones científicas ${ }^{1}$, y se tomaron en consideración atributos métricos. Las medidas de los especimenes paleontológicos fueron tomadas siguiendo los criterios de von den Driesch (1999), Cartajena (2002) y Alberdi y Prado (2004), los que fueron comparados con datos de la literatura (Alberdi y Prieto 2000; Cartajena 2002; Menegaz 2000; Alberdi datos sin publicar) y obtenidos de primera fuente.

La estimación de edad de los restos analizados se basa en las tablas de fusión epifisiaraia de Kauffman (2004) para Lama guanicoe Muller, Purdue (1983; cfr. Mena 1992) para Odocoileus virginianus Zimmerman y en la tabla de erupción dental de Equus caballus Linnaeus de Levine (1982). La falta de información directa al respecto sobre parte de las especies que nos competen justifica recurrir a taxones afines.

1 Departamento de Antropología de la Universidad de Chile, Laboratorio de Arqueología de la Universidad Bolivariana, Laboratorio de Arqueología de la Universidad Internacional SEK, Museo Nacional de Historia Natural de Chile, Centro de Estudios del Hombre Austral-Universidad de Magallanes y Museo de La Plata (Argentina). 
La cuantificación de los restos se expresa en términos de NISP, o número de especimenes identificados y MNE o número mínimo de elementos. Para efectos del análisis de la frecuencia de partes esqueletarias, los distintos elementos que componen la carcasa fueron agrupados tomando en consideración su ubicación en el esqueleto y propiedades intrínsecas (cfr. Stiner 1991). Su variación dentro de los conjuntos faunísticos estudiados se representa en función de la relación MNE observado/MNE esperado. En este trabajo, las unidades analíticas utilizadas tienen como base el esqueleto de los artiodáctilos, ya que corresponden al grupo taxonómico más representado. Estas se dividen en: cráneo (cráneo y dos hemimandíbulas, MNE:3); axial (vértebras cervicales, vértebras torácicas, vértebras lumbares y sacro, MNE:27); costillas (MNE:24); extremidades superiores (escápulas, húmero, coxales, fémures, MNE:8); extremidades medias (radioulnas y tibias, MNE:4); extremidades distales (tarsos, carpos y metapodios, MNE: 32) y pies (falanges primeras, segundas y terceras, MNE:24). Los distintos taxones identificados se agruparon en tres categorías de acuerdo a su peso: Grupo 1 (0-70 Kg), Grupo 2 (71$150 \mathrm{Kg}$ ) y Grupo 3 (más de $150 \mathrm{Kg}$ ). Se utilizaron adicionalmente datos comparativos de Borrero et al. (1997), Marean y Ehrhardt (1995), y Martin y Borrero (1997).

En el análisis tafonómico efectuado se consideraron la meteorización (Behrensmeyer 1978), pisoteo, pulimento, abrasión (Beherensmeyer et al. 1986), modificaciones por acción de carnívoros (Binford 1981; Mondini 2003), marcas de raíces, (Lyman 1994) y huellas de ácidos digestivos de acuerdo a las categorías de Schmitt y Juell (1994), utilizando la escala de intensidad sugerida por
Andrews (1990). En lo que respecta a las modificaciones culturales se consideraron huellas de corte, percusión, machacado y raspado (Mengoni 1999), así como atributos tecnológicos relacionados con la confección de instrumentos (Eiroa et al. 1999).

\section{RESULTADOS}

\section{Identificación taxonómica}

La muestra analizada se caracteriza por su elevada fragmentación, lo que se tradujo en una baja frecuencia de elementos identificados (NISP:101; $6,37 \%$ del total, excluyendo a los osteodermos). El análisis taxonómico indica la presencia de formas tanto extintas como vivientes (Tabla 1). Entre las primeras se incluyen Hippidion saldiasi Roth 1899 , representado por 12 restos dentales, en los que destacan cuatro molares deciduos (dos dPM4 derechos, dPM2 izquierdo, dPM indet.), los que a juzgar por la erupción dental de Equus caballus dan cuenta de dos individuos menores de tres años y medio (Levine 1982) (Fig. 3a). Los molares permanentes (PM2 derecho, M2 izquierdo, M1-3, M indet, PM3-4 derecho y M2 indet.), por su parte, indican la presencia de un individuo adulto maduro (mayor de cinco años, Levine 1982). Son particularmente diagnósticos del género los molares superiores permanentes ya que presentan una morfología general cuadrangular, los protoconos de forma oval-redondeada, ausencia de pliegue caballino y de pliegues en las prefosetas y postfosetas. La asignación específica se logró a través de criterios métricos ya que $H$. saldiasi corresponde a la especie más pequeña de hippidiformes hacia el Pleistoceno final en Sudamérica (Alberdi y Prado 2004). La Fig. 4a, grafica el largo mesiodistal/ancho

TABLA 1. Resumen de restos analizados en las capas basales de cueva Las Guanacas.

\begin{tabular}{llcccccc}
\hline & & Capa 9 & Capa 10 & Capa 11 & Capa 12 & Sin Inf. & Total \\
\hline Camelidae & Lama guanicoe & - & 7 & - & - & - & 7 \\
Cervidae & Camelidae indet. & 3 & 2 & - & 1 & 2 & 8 \\
& Hippocamelus bisulcus & - & 5 & - & - & - & 5 \\
Artiodactyla mediano & Pudu pudu & - & 4 & - & - & - & 4 \\
Equidae & Hippidion saldiasi & - & 31 & 1 & 11 & - & 43 \\
Macraucheniidae & Macrauchenia patachonica & - & 5 & - & 2 & 5 & 12 \\
Mylodontidae & Mylodon darwini & 30 & 479 & 18 & 82 & 2 & 611 \\
Canidae & Dusicyon culpaeus & - & 6 & 3 & 12 & - & 21 \\
Mammalia & & 5 & 755 & 11 & 102 & - & 873 \\
& Total & 38 & 1295 & 33 & 210 & 9 & 1585 \\
\hline
\end{tabular}


a.

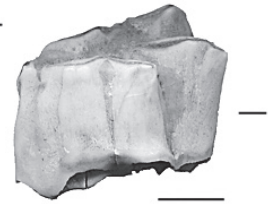

d.

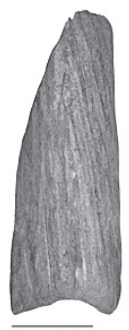

b.

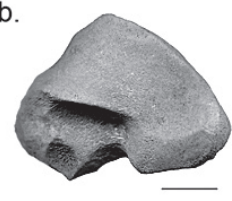

e.
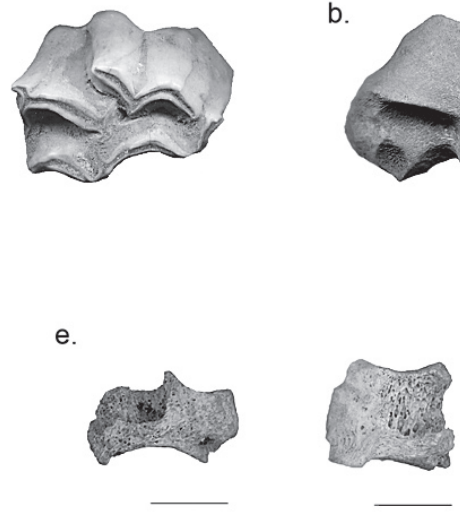

C.

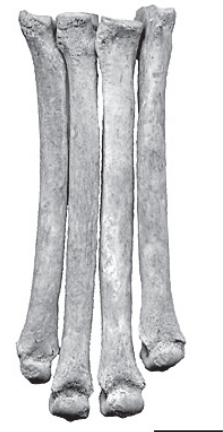

Fig. 3. a. dPM4 de Hippidion saldiasi, vistas lingual y oclusal; b. cuarto carpiano de Camelidae indet., vista proximal; c. metatarsos II-V de Dusicyon culpaeus; d. fragmento de instrumento confeccionado sobre artiodáctilo indet.; e. huesos cortos con huellas de ácidos digestivos. La barra mide $1 \mathrm{~cm}$.

bucolingual/largo del protocono del M2, en donde se aprecia un tamaño menor que $H$. principale (Apéndice 1, Tabla 1).

De igual manera, se identificaron dentro de la muestra de cueva Las Guanacas restos óseos de morfología compatible con la familia Camelidae pero de tamaño mayor a las observadas en las formas vivientes (Fig. 3b; Apéndice 1, Tabla 2). La Fig. 4b muestra similitudes métricas con las especies extintas Palaeolama (Hemiauchenia) paradoxa Gervais y Ameghino (sensu Guerin y Faure 1999) y Lama owenii Gervais y Ameghino (sensu Menegaz 2000) a nivel de esqueleto postcraneal. Lamentablemente, los caracteres diagnósticos se concentran fundamen- talmente en el cráneo y mandíbula (Cabrera 1932; Scherer et al. 2007), por lo que no fue posible realizar asignaciones taxonómicas más precisas.

El abundante registro de huesecillos dérmi$\cos (\mathrm{N}: 611)$ recuperados fue asignado a Mylodon darwini Owen 1839, debido principalmente a dos criterios. En primer término, la monoespecificidad del género hacia el finipleistoceno de acuerdo a la revisión de los Mylodontinae de Esteban (1996), y a la ausencia en el área de otros taxones con osículos dérmicos como Glossotherium robustum Owen, Eremotherium laurillardi Lund o Glyptodon clavipes Owen (Cartelle y Bohórquez 1986; Esteban 1996, Labarca 2008; Rinderknecht 2000).
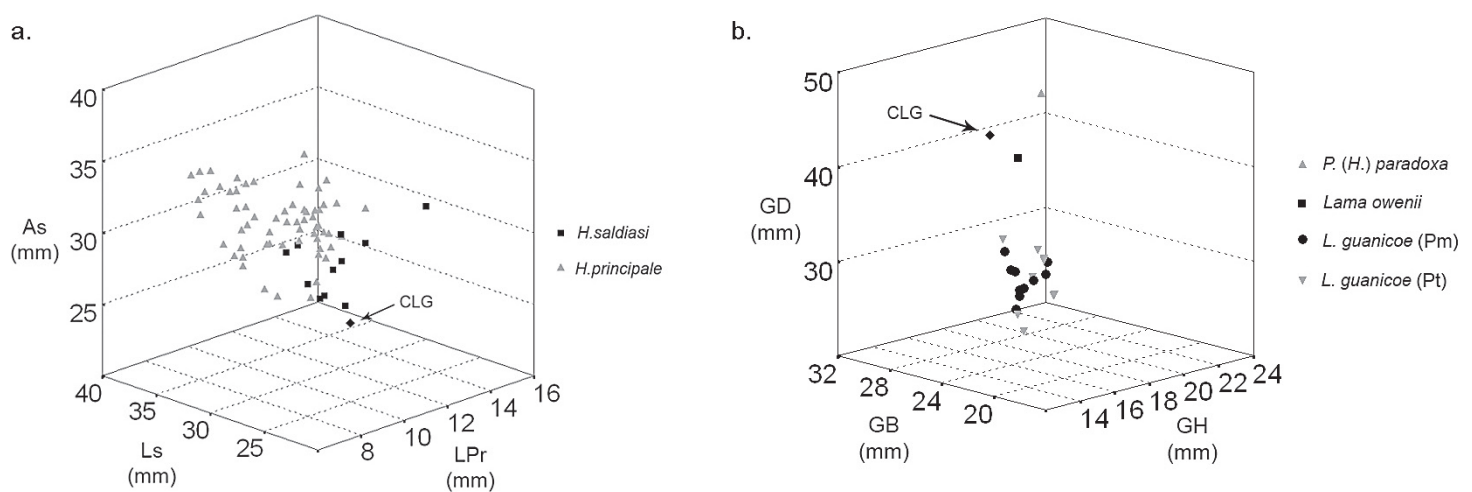

Fig. 4. a. Dispersión de medidas de M2 de hippidiformes del Pleistoceno final sudamericano; b. Dispersión de medidas de cuarto carpiano de distintos integrantes de la familia Camelidae. Pm: pampa; Pt: Patagonia; CLG: cueva Las Guanacas. Abreviaturas según Apéndice 1. 
El último taxón extinto corresponde a $\mathrm{Ma}$ crauchenia patachonica Owen 1939, identificado a partir de una porción proximal de una primera falange (Apéndice 1, Tabla 3). Esta asignación se logró mediante la comparación directa con esqueletos de referencia.

La elevada presencia de restos postcraneales de cánidos (Tabla 2), algunos de los cuales articulan (Fig. 3c), abría la posibilidad de detectar alguna especie de zorro extinto (p.e Dusicyon avus Burmeister), teniendo en consideración los antecedentes del vecino yacimiento de Baño Nuevo (Trejo y Jackson 1998). Debido a que los caracteres diagnósticos que diferencian a las formas vivientes de las extintas dentro del género Dusicyon se concentran en las series dentales (p.e mayor longitud del molar carnicero, un hipoconúlido en el talónido del M1), la asignación taxonómica de los restos se basó en el supuesto del mayor tamaño relativo y robustez que habría tenido $D$. avus (Berman y Tonni 1986; Prevosti y Vizcaíno 2006). En este caso, se tomaron como referencia esqueletos actuales de Dusicyon culpaeus Molina de la Región de Magallanes y del Maule, y de D. griseus Molina de la Región Metropolitana. Como se aprecia en la
Fig. 5, los especimenes de cueva de Las Guanacas corresponderían a D. culpaeus Molina 1789, el que presentaría un menor tamaño que aquel registrado en Patagonia Austral (Apéndice 1, Tabla 4).

El registro paleofaunístico se completa con taxones pertenecientes al orden Artiodactyla: Lama guanicoe Muller 1776, Hippocamelus bisulcus Molina 1789 y Pudu pudu Molina 1789 (Apéndice 1). De estos últimos dos no existían registros pleistocénicos. La presencia de algunas epífisis sin fusionar en el caso del guanaco apunta hacia un individuo juvenil, el que a juzgar por la tabla de fusión propuesta por Kauffman (2004) habría tenido menos de 34 meses al momento de su muerte. Respecto del huemul, teniendo como referencia los estadios de fusión de su cercano pariente Odocoileus virginianus (Purdue 1983), se estaría en presencia de un individuo menor de 20 meses. Por último, los restos de pudú dan cuenta de dos individuos: un adulto joven y uno maduro. Finalmente se consignó la categoría Artiodactyla mediano para designar todos aquellos restos no identificados taxonómicamente con un tamaño compatible con guanaco/huemul (Tabla 2).

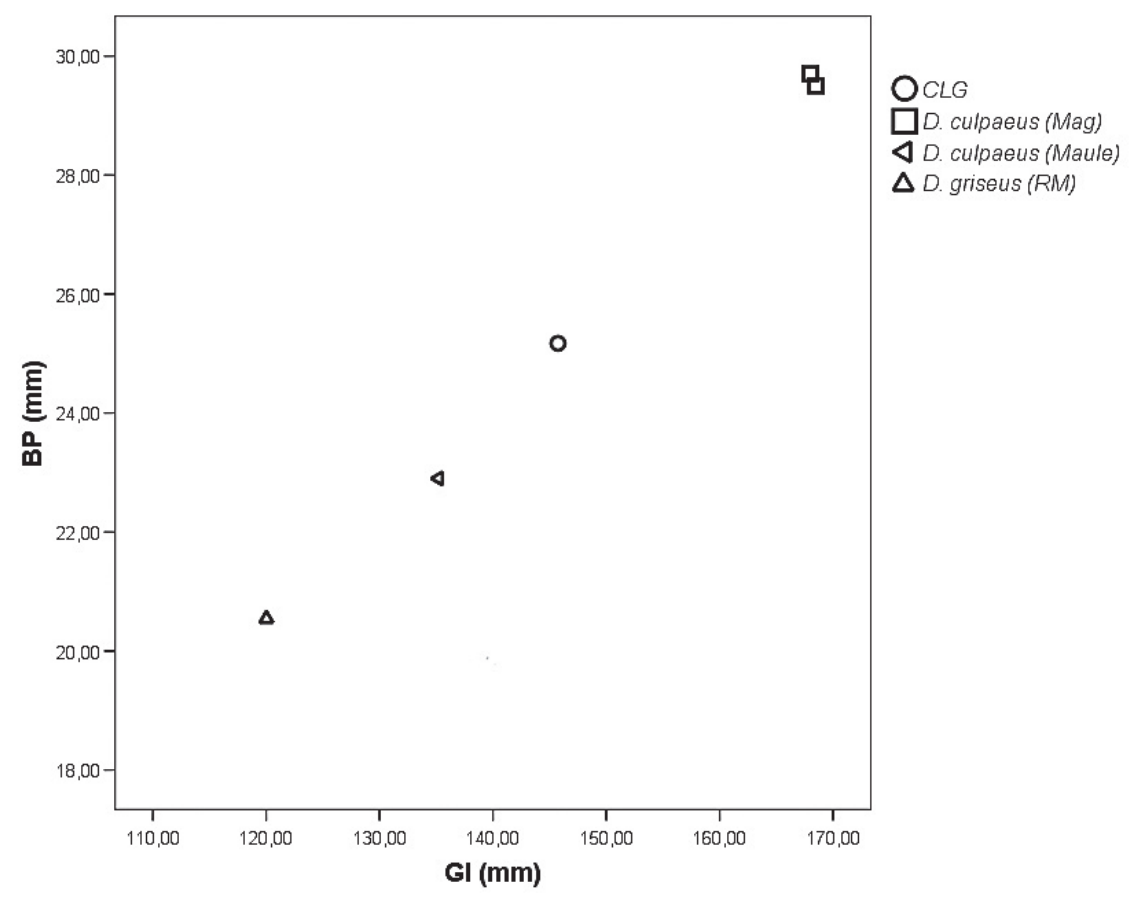

Fig. 5. Dispersión de medidas de tibias de Dusicyon. Mg: Magallanes; CLG: cueva Las Guanacas; RM: región Metropolitana. Abreviaturas según Apéndice 1. 
TABLA 2. Resumen de unidades anatómicas identificadas en las capas basales de cueva de las Guanacas. Se excluyen los restos de H. saldiasi y M. patachonica descritos en el texto.

\begin{tabular}{|c|c|c|c|c|c|c|c|c|c|c|c|c|}
\hline & \multicolumn{2}{|c|}{ L. guanicoe } & \multicolumn{2}{|c|}{ P. pudu } & \multicolumn{2}{|c|}{ H. bisulcus } & \multicolumn{2}{|c|}{$\begin{array}{l}\text { Artiodactyla } \\
\text { mediano }\end{array}$} & \multicolumn{2}{|c|}{ Camelidae } & \multicolumn{2}{|c|}{ D. culpaeus } \\
\hline & NISP & MNE & NISP & MNE & NISP & MNE & NISP & MNE & NISP & MNE & NISP & MNE \\
\hline Molar Sup. & 1 & 1 & 1 & 1 & - & - & - & - & - & - & - & - \\
\hline Molar Inf & - & & 1 & 1 & - & & 1 & 1 & 2 & 2 & & \\
\hline Molar Indet. & - & - & - & - & - & - & 3 & 1 & 1 & 1 & - & - \\
\hline Incisivo & - & - & - & - & - & - & - & - & - & - & - & - \\
\hline V. Cervical & - & & - & & - & & 2 & 2 & - & & & \\
\hline V. Lumbar & - & - & - & - & 1 & 1 & - & - & - & - & - & - \\
\hline V. Indet. & - & - & - & - & - & - & 7 & 2 & - & - & - & - \\
\hline Sacro & - & - & - & - & - & - & 1 & 1 & - & - & - & - \\
\hline Costilla & - & - & - & - & - & - & 5 & 2 & - & - & 1 & 1 \\
\hline Escápula & - & & & & & & 1 & 1 & & & 3 & 3 \\
\hline Húmero & - & - & - & - & - & - & - & - & - & - & 2 & 2 \\
\hline Radioulna & - & - & - & - & 1 & 1 & - & - & - & - & - & - \\
\hline Carp. Ulnar & 1 & 1 & - & & - & & - & & - & & & \\
\hline Carp.Radial & 1 & 1 & - & - & - & - & - & - & - & - & - & - \\
\hline Carpo 2 & - & - & - & - & - & - & - & - & 1 & 1 & - & - \\
\hline Carpo 4 & - & - & - & - & - & - & - & - & 1 & 1 & - & - \\
\hline Metacarpo & - & - & - & - & - & - & - & - & - & - & 4 & 4 \\
\hline Coxal & & - & 1 & 1 & & & 1 & 1 & 2 & 1 & & \\
\hline Baculum & - & - & - & - & - & - & - & - & - & - & 1 & 1 \\
\hline Fémur & - & - & 1 & 1 & 1 & 1 & 1 & 1 & 1 & 1 & - & - \\
\hline Patela & 1 & 1 & - & & - & & & & & & & \\
\hline Tibia & - & - & - & - & 2 & 2 & - & - & - & - & 1 & 1 \\
\hline Fíbula & - & - & - & - & - & - & - & - & - & - & 1 & 1 \\
\hline Maleolo Lat & - & - & - & - & - & - & 1 & 1 & - & - & - & - \\
\hline Astrágalo & - & - & - & - & - & - & - & - & - & - & 1 & 1 \\
\hline Calcáneo & & - & & - & - & - & - & - & - & - & 1 & 1 \\
\hline Metatarso & - & - & - & - & - & - & - & - & - & - & 4 & 4 \\
\hline Metapodio & - & - & - & - & - & - & 2 & 2 & - & - & - & - \\
\hline Falange 1 & 2 & 2 & & & & & & & & & 1 & 1 \\
\hline Falange 2 & 1 & 1 & - & - & - & - & - & - & - & - & 1 & 1 \\
\hline Falange 3 & - & - & - & - & - & - & 1 & 1 & - & - & - & - \\
\hline Falange Ind. & - & & - & & - & & 1 & 1 & & & & \\
\hline Total & 7 & 7 & 4 & 4 & 4 & 4 & 27 & 17 & 8 & 7 & 21 & 21 \\
\hline
\end{tabular}

Tafonomía

Como se mencionó anteriormente, el conjunto analizado en las capas basales de cueva Las Guanacas exhibe en general una elevada fragmentación, lo que se tradujo en una baja identificación (6,37\%). El grueso de los especimenes no identificados taxonómicamente (Mammalia indet. y Artiodactyla mediano) corresponden a astillas y fragmentos de tejido trabecular con un largo promedio de $22,4 \mathrm{~mm}$, a lo que se suman algunas fracciones de vértebras y costillas. Esta situación contrasta con lo observado en las capas holocénicas de la cueva, donde los restos identificados en el bloque tardío y medio, alcanzan un $79,87 \%$ y $83,23 \%$, respectivamente (Fuentes y Mena 2008).

Las observaciones tafonómicas realizadas, denotan en general una muy baja proporción de marcas naturales (Tabla 3), identificándose fundamentalmente especimenes con huellas dendríticas, evidencias de pulimento (producto de ceniza volcánica, en muchos casos adosada a los huesos), acción de roedores y carnívoros. 
La acción de roedores y de carnívoros en cueva Las Guanacas se define por la presencia de punctures, scoring, furrowing (Binford 1981) y corrosión digestiva. Las primeras categorías se encuentran constatadas sobre fragmentos no identificados (N:9), vértebras de un mamífero juvenil no determinado $(\mathrm{N}: 3)$ y un coxal juvenil de Camelidae indet. (N:1). A juzgar por las piezas con marcas, la ubicación de éstas y la intensidad del consumo, es posible postular como agente responsable a un carnívoro de pequeño tamaño, posiblemente un cánido. Comparativamente, las huellas de corrosión digestiva son más abundantes (50,78\% del total de las modificaciones, Tabla 3), registrándose fundamentalmente la categoría leve (56,9\%) (Schmitt y Juell 1994, Andrews 1990). Aunque en menor proporción (9,23\%), se destaca la presencia de unidades óseas con una intensa acción de ácidos gástricos, las que incluso implican la disolución de una porción significativa del tejido esponjoso (Fig. $3 e)$. Este atributo se observa en huesecillos dérmicos de M. darwini ( $\mathrm{N}: 25)$, como también en astillas, fragmentos de porciones epifisiales y huesos cortos de Mammalia indet. (N:37), Artiodactyla indet.(N:1), Lama guanicoe (N:1) y Camelidae indet. (N:1). Estas últimas permitirían plantear la existencia de un segundo carnívoro, de talla mediana o grande, a juzgar por la intensidad de la acción de los agentes digestivos y las dimensiones de algunos especimenes con este tipo de marcas. Este punto será discutido en el siguiente acápite.

Las huellas de carácter cultural, se encuentran completamente ausentes en la muestra, detectándose de forma excepcional sólo una pieza que podría sugerir una efímera ocupación temprana en el sitio. Se trata de un artefacto elaborado sobre una fracción de diáfisis (11B-N14-C10), posiblemente la porción medial de una tibia, perteneciente a un artiodáctilo mediano ( $H$. bisulcus o L. guanicoe). Su superficie se encuentra pulida naturalmente por la acción de cenizas presentes en la matriz del sitio, mostrando semejanza con algunos especimenes de capas adyacentes con símiles cualidades (Tabla 3). Se distingue de los ecofactos por la presencia de ranurados longitudinales presentes en ambos flancos de la diáfisis, los que se emplazan en la cara externa, y que además convergen hacia el vértice angular del hueso (Fig. 3d).

\section{DISCUSIÓN}

Tomando en conjunto las características de la muestra analizada, no existe evidencia sólida que permita respaldar una ocupación humana en cueva Las Guanacas anterior al 5.000 AP. En efecto, la presencia de una porción de artefacto de pequeñas dimensiones en las capas finiplesitocénicas puede deberse a migraciones verticales ocurridas posterior a la desocupación del yacimiento, situación que ha sido documentada en otros asentamientos patagónicos (p.e Tres Arroyos, Massone 2004), así como en conjuntos tafonómicos actuales (Borrero 1990). El registro microfaunístico de Las Guanacas incluye una importante cantidad de roedores fosoriales (p.e Ctenomys sp.), y aunque algunos de éstos pudieron haber ingresado a través de egagrópilas de aves rapaces o fecas de carnívoros, la presencia de negativos de incisivos en algunos especimenes óseos indican que la cueva fue habitada por fauna cavícola, los que eventualmente pudieron realizar algún tipo de disturbación estratigráfica no perceptible (p.e ma-

TABLA 3. Resumen (NISP) de modificaciones naturales en las capas pleistocénicas de cueva Las Guanacas.

\begin{tabular}{lcccccc}
\hline Taxa & Raíces & Carnívoros & Roedor & H. ácido & Pulido & Total \\
\hline Lama guanicoe & - & - & - & 1 & - & 1 \\
Camelidae indet. & - & 1 & - & 1 & - & 2 \\
Hippocamelus bisulcus & - & - & - & - & 1 & 1 \\
Pudu pudu & 1 & - & - & - & - & 1 \\
Artiodactyla mediano & 1 & 2 & - & 1 & 1 & 5 \\
Hippidion saldiasi & - & - & - & - & - & - \\
Mylodon darwini & - & - & 1 & 25 & - & 26 \\
Macrauchenia patachonica & - & - & - & - & 1 & 1 \\
Dusicyon culpaeus & 6 & - & 5 & - & 8 & 19 \\
Mammalia & - & 10 & - & 37 & 25 & 72 \\
Total & 8 & 13 & 6 & 65 & 36 & 128 \\
\hline
\end{tabular}


drigueras colapsadas). Esta interpretación se vería apoyada por la presencia de huesecillos dérmicos de Mylodon darwini en las capas más tardías del yacimiento, asociados directamente a las ocupaciones humanas (Fuentes y Mena 2008).

Descartando una ocupación cultural finipleistocénica en cueva Las Guanacas, resulta interesante discutir cuál habría(n) sido el(los) agente(s) que acumuló(aron) los restos óseos. Desechamos muertes naturales de los herbívoros recuperados al interior de la cueva, ya que la etología de éstos no es compatible con la utilización de reparos, más aún considerando la ubicación del abrigo (150 m sobre el nivel de la terraza fluvial) y su difícil acceso. La única excepción la conforma Mylodon darwini, el que habría ocupado reparos rocosos como habitación (Bird 1993). No obstante éste edentado se encuentra representado exclusivamente por osteodermos. Una primera revisión a las modificaciones naturales presentes apuntan a hacia un carnívoro (mordeduras y ácidos digestivos). Hipotetizar qué taxón(es) fue(ron) y qué estrategia de obtención de las presas habría(n) implementado es el tema central de la discusión que sigue. Para desarrollar esta, se tomaron en cuenta la etología de los depredadores del área hacia el Pleistoceno final, ubicación espacial de los restos en la cueva, presas presentes, frecuencia de partes esqueletarias, huellas de mordeduras, incidencia de ácidos digestivos y tamaño de los restos con este tipo de modificaciones.

Hacia el finipleistoceno en Patagonia austral el registro fósil apunta hacia un complejo ensamble de depredadores, los que en conjunto con el variado elenco de mamíferos herbívoros habrían conformado una rica cadena trófica (cfr. Prevosti y Vizcaíno 2006). El registro paleofaunístico incluye por lo menos seis especies de depredadores con hábitos mas o menos carnívoros: Smilodon populator Lund, Panthera onca Linnaeus, Arctotherium tarijenese Ameghino, Puma concolor Linnaeus, Dusicyon avus y Dusicyon culpaeus (p.e Caviglia 1985-1986; Labarca 2008; Prevosti et al. 2003). Si bien hasta ahora el registro de carnívoros pleistocénicos de Patagonia central chilena indica sólo la presencia de los dos últimos, las afinidades faunísticas con Patagonia austral en términos de mamíferos herbívoros permitirían sustentar la presencia de depredadores de mayor tamaño, como los cuatro primeros de la lista. Esta cuestión ha sido indirectamente sostenida en la cueva de Baño Nuevo a partir de análisis exclusivamente tafonómicos (López 2008). Estudios de corte actualístico han demostrado que los zorros actuales trasladan unidades anatómicas de presas de tamaño medio a sus madrigueras (p.e camélidos y caprinos), las que aparentemente obtienen a partir del carroñeo (Mondini 1995, 2003), por lo que es posible sugerir que $D$. avus podría haber implementado una estrategia similar. Muy poco se sabe respecto de la etología de los osos del género Arctotherium y no es posible asegurar a priori que habrían utilizado cuevas como dormideros, ya que por ejemplo, Tremarctos ornatus Cuvier no hiberna (Peyton 1980). El hecho de que se hayan recuperado restos de $\mathrm{A}$. tarijense en cueva del $\mathrm{Mi}$ lodón (Última Esperanza) y Cueva del Puma (Pali Aike) podría indicar que este úrsido habría utilizado reparos rocosos (Martin et al. 2004; Prevosti et al. 2003). Respecto de los félidos, tradicionalmente se ha sugerido que los carnívoros de hábitos solitarios no utilizan madrigueras de manera regular para su alimentación (Martin y Borrero 1997). Por ejemplo, el puma consume sus presas en lugares cercanos al sitio de matanza, cubriendo las carcasas con materia vegetal, para así asegurar que otros predadores menores no accedan a ella (Iriarte et al. 1991; Quintana et al. 2000). Sin embargo, bajo ciertas situaciones estas conductas pueden variar, como por ejemplo cuando la competencia inter e intra específica es muy fuerte, en la época de parición o en momentos de estrés (Martin y Borrero 1997; Stiner 1991; Turner y Antón 1997). Martin y Borrero (1997) documentan y estudian en detalle una madriguera de puma en Patagonia austral, la que habría sido utilizada por una hembra durante el proceso de alimentación de las crías. De igual manera Marean y Ehrhardt (1995), analizan los restos fósiles recuperados de una madriguera de Homotherium serum Fabrini (parte de la subfamilia de los gatos dientes de sable) en donde los individuos juveniles de este Felidae alcanzan a un 39,39\% de la muestra. De manera indirecta, y tomando como referencia los ecosistemas africanos conformados por un gran número de carnívoros, es posible sugerir que la utilización de reparos hacia el final del Pleistoceno en Patagonia debió ser mucho más intensa que ahora, debido a la mayor competencia generada por el complejo ensamble de carnívoros documentados en el área. En la actualidad Pu. concolor es el mayor carnívoro 
que habita la región por lo que difícilmente podrá verse amenazado durante el proceso de consumo de sus presas. Hace 13.000 años atrás $\mathrm{Pu}$. concolor ocupaba un lugar secundario dentro de los consumidores terciarios, por lo que la utilización de refugios para una alimentación segura parece una respuesta adaptativa esperable. Esta hipotética situación es también válida para $P a$. onca, ya que habría competido con $\mathrm{Pu}$. concolor y A. tarijense por mamíferos de tamaño medio (Prevosti y Vizcaíno 2006). De lo anterior se desprende que cueva Las Guanacas podría haber sido eventualmente ocupada por cualquiera de los carnívoros mencionados.

Una vía para poder definir el o los posibles depredadores que habrían utilizado cueva Las Guanacas es realizar comparaciones con estudios tafonómicos actualísticos y paleontológicos de madrigueras de los distintos carnívoros potenciales. De acuerdo a la exhaustiva revisión de Cleghorn y Marean (2007) gran parte de los trabajos en madrigueras de carnívoros adolecen de una falencia grave, ya que no es posible acceder al universo total de unidades trasladadas, debido a que las piezas que efectivamente se recuperan en los refugios se encuentran mediadas por los procesos de consumo de los depredadores. Por ejemplo, hienas y cánidos destruyen e ingieren preferentemente aquellas unidades óseas menos densas pero ricas en grasa (p.e epífisis), por lo que las frecuencia de partes que surge del estudio de madrigueras se encuentra sesgada a favor de las unidades más densas y menos atractivas (p.e diáfisis). No obstante lo anterior, se debe tener en cuenta que la gran mayoría de los trabajos actualísticos conducidos han sido realizados con animales que poseen una morfología dental preparada para el consumo de huesos (p.e presencia de mayor número de molares y premolares, mayor superficie oclusal en éstos, [Turner y Antón 1997]) (Binford 1981; Brain 1981; Haynes 1983, entre otros). Ciertos depredadores, principalmente félidos, poseen una dieta mucho más carnívora con una morfología dental orientada al consumo de carne por sobre hueso (p.e ausencia de talónido en el m1 y de molares postcarniceros, presencia de molares carniceros del tipo secodonte, escasa superficie oclusal en los molares [Prevosti y Vizcaíno 2006]). Al consumir comparativamente una menor cantidad de restos óseos, las frecuencias de partes anatómicas reflejaran de mejor manera las decisiones de transporte de estos animales. Una consecuencia que se colige de esta conducta, es que los félidos dejarán comparativamente menos marcas asociadas al consumo de huesos en las carcasas que aquellos que incluyen los restos óseos de manera más regular en la dieta.

Las modificaciones por carnívoros registradas en cueva Las Guanacas se relacionan en tres casos directamente con un carnívoro de tamaño pequeño, lo que permite un primera aproximación a los agentes depositacionales que habrían actuado en sitio. No obstante, su incidencia en el conjunto es baja ( $0,8 \%$ de los restos), lo que no apuntaría hacia un conjunto generado exclusivamente por cánidos. Más aún, se registran epífisis proximales y distales de hueso largos, así como vértebras y costillas sin huellas de mordeduras (Tabla 3), lo que sugeriría un depredador que consume preferentemente carne por sobre hueso. A esto debemos agregar el hecho de que existe una relación entre el peso del predador y su presa (Radlof y Du Toit 2004), por lo que difícilmente un zorro colorado habría podido cazar taxones extintos cuya masa supera largamente en muchos casos los 200 Kg (Fariña et al. 1998; Prado y Alberdi 2004; Prevosti y Vizcaíno 1996). Aún si pudiera carroñear sobre ellos y trasladarlos a su cubil, es esperable una mayor proporción de marcas de consumo, así como una frecuencia de partes esqueletales dominada por porciones distales de las extremidades (Mondini 1995, 2003), situación que no se registra en cueva Las Guanacas (ver más abajo). En nuestra opinión $D$. culpaeus (u otro taxón afín) habría carroñeado al interior del cueva los restos dejados por otro predador, aunque no es posible descartar que haya ingresado algunas porciones anatómicas, particularmente aquellas de animales más pequeños (p.e pudú). En este sentido, la presencia de restos óseos de D. culpaeus in situ, aun posibles de articular, es una cualidad identificada en dormideros, y excepcionalmente en letrinas, al menos para el caso del género Dusicyon de regiones relativamente próximas (p.e. Lago Argentino y Torres del Paine) (Martin 1998).

De esta manera, la baja frecuencia de marcas de carnívoros sumado a la presencia de unidades menos densas y ricas en nutrientes atractivas para carroñeros adaptados al consumo de huesos, sugieren, en principio, un contexto generado por un félido por sobre un cánido. Aún cuando los trabajos de tafonomía de úrsidos son comparativamente 
menores es posible sugerir una conducta relativamente similar a la mencionada para los cánidos, en donde las marcas generadas por el consumo de unidades óseas son evidentes y diferenciables (Haynes 1983). En este sentido, Soibelzon (2002 en Prevosti y Vizcaíno 2006) indican que la morfología dental de Arctotherium se encontraba preparada para fracturar huesos, aunque habría tenido una dieta omnívora.

Por otro lado, no de deja de ser sugerente el hecho de que la gran mayoría de los restos de ammíferos extintos provengan de cuadrículas ubicadas al fondo de la cueva (Labarca, datos sin publicar), ya que se ha documentado que los felinos prefieren lugares oscuros para el establecimiento de sus madrigueras (Turner y Antón 1997). La información faunística proveniente de las cuadrículas excavadas en el frente de la cueva, indica comparativamente menos restos de mamíferos extintos, correspondiendo éstos en su gran medida a huesecillos dérmicos, por lo que es posible postular que éste sector habría sido menos atractivo para los predadores finipleistocénicos. La preferencia de los félidos por lugares con baja luminosidad ha sido confirmada en nuestra área de estudio, por la presencia de cubiles de pumas actuales en el campo de lava de Pali Aike y guaridas de félidos extintos en Última Esperanza (Borrero et al. 1997; Martin et al. 2003) que además son de difícil acceso, como en cueva Las Guanacas.

Prevosti y Vizcaíno (2006) calcularon los tamaños de presa máximos y típicos para los carnívoros pampeanos, los que se han documentado igualmente en el área patagónica. Descartando cánidos y úrsidos, acá presentaremos los datos obtenidos para félidos. $S$. populator habría predado sobre taxones con una masa máxima de $1871,56 \mathrm{Kg}$, con una presa típica de $764,16 \mathrm{Kg}, \mathrm{Pa}$. onca habría optado por presas con una masa de hasta $564,62 \mathrm{Kg}$, pero su presa típica habría rondado en $135,68 \mathrm{Kg}$. Estos cálculos son superiores a los registrados actualmente, en donde el jaguar preda preferentemente sobre el capibara (Hydrochoerus hydrochoeris Linnaeus) (45 Kg) y pecarí (Tayassu sp.) $(25,5 \mathrm{Kg})$, aunque accede de manera regular al ganado doméstico (ca. $291 \mathrm{Kg}$ ) (Cascelli de Acevedo y Murria 2007; Palmeira et al. 2008; Garla et al. 2001). Probablemente estas diferencias se deban al supuesto mayor tamaño del jaguar pleistocénico. Pu. concolor, predaría de manera principal sobre individuos de $26,9 \mathrm{Kg}$, pero podría acceder a taxones de hasta $500 \mathrm{Kg}$. (Prevosti y Vizcaíno 2006). Estudios realizados en Patagonia indican en términos generales una dieta dominada por mamíferos como L. guanicoe, $H$. bisulcus, Pudu pudu y fauna introducida (Ovis aries Linnaeus y Lepus capensis Linnaeus) (Franklin et al. 1999). No obstante, investigaciones desarrolladas en Torres del Paine (Región de Magallanes) indican una dieta compuesta principalmente por liebre (Lepus capensis) (ca. $3 \mathrm{Kg}$ ), aun cuando el consumo de guanaco (principalmente juvenil, ca. $50 \mathrm{Kg}$.) habría aumentado en el último tiempo (Iriarte et al. 1991; Franklin et al. 1999). Resulta interesante destacar que los estudios sobre predación del ganado bovino por parte del puma y el jaguar en Brasil demuestran que el primero caza de preferencia individuos de hasta 3 meses $(50 \mathrm{Kg})$, mientras que el segundo centra sus actividades en torno a aquellos de 3 y 9 meses de edad (hasta $180 \mathrm{Kg}$ ). Investigaciones con fauna autóctona han arribado similares conclusiones (Scognamillo et al. 2003). Se debe tener presente que la relación del tamaño predador-presa no implica que los carnívoros de mayor tamaño se alimentan exclusivamente de las presas más grandes, sino que éstos simplemente tienen un abanico de posibilidades mayor (Radlof y Du Toit 2004). Contrario a esto, se ha argumentado que los "gatos dientes de sable" habrían evolucionado hacia una especialización en mamíferos de gran tamaño (Berta 1987). Estudios en una madriguera de Homotherium en el sur de Estados Unidos indican una sobre representación de proboscídeos juveniles, lo que apoyaría esta última impresión (Marean y Ehrhardt 1995).

Los taxones identificados en la muestra de cueva de Las Guanacas incluyen Pudu pudu (10 $\mathrm{Kg})$, L. guanicoe (90 Kg), Hippocamelus bisulcus $(120 \mathrm{Kg})$, Hippidion saldiasi (ca. $250 \mathrm{Kg})$, Ma. patachonica $(988 \mathrm{Kg})$, Camelidae indet. (1000 Kg para Palaeolama (H.) paradoxa) y My. darwini $(1000 \mathrm{Kg})$ (Alberdi y Prado 2004; Fariña et al. 1998; González et al. 2000; Prevosti y Vizcaíno 2006). De acuerdo a los datos mencionados en el párrafo anterior, es posible postular que tanto el puma como el jaguar habrían predado sobre los artiodáctilos de tamaño pequeño y medio, y sólo ésta última podría haber accedido de manera más regular a ejemplares de caballo americano. S. populator, por el contrario, no habría tenido inconvenientes para cazar todos los taxones representados en cueva Las Guanacas, 
TABLA 4. Resumen de unidades anatómicas identificadas por grupos de peso.

\begin{tabular}{llccccccccc}
\hline & & \multicolumn{3}{c}{ Grupo 1 } & \multicolumn{3}{c}{ Grupo 2 } & \multicolumn{3}{c}{ Grupo 3 } \\
\hline \multirow{3}{*}{ Cráneo } & & NISP & MNE & MNI & NISP & MNE & MNI & NISP & MNE & MNI \\
& Molar Sup & 1 & 1 & 1 & 1 & 1 & 1 & 4 & 4 & 3 \\
& Molar Inf & 1 & 1 & 1 & 1 & 1 & 1 & 8 & 7 & 1 \\
& V. Cervical & - & - & - & 2 & 2 & 1 & - & - & - \\
& V. Torácica & - & - & - & - & - & - & - & - & - \\
& V. Lumbar & - & - & - & 1 & 1 & 1 & - & - & - \\
\multirow{5}{*}{ Costillar } & Sacro & - & - & - & 1 & 1 & 1 & - & - & - \\
\multirow{3}{*}{ Ext. Proximal } & V Indet. & - & - & - & 7 & 2 & 1 & - & - & - \\
& Costilla & - & - & - & 5 & 2 & 1 & - & - & - \\
\multirow{5}{*}{ Ext. Media } & Escápula & - & - & - & 1 & 1 & 1 & - & - & - \\
\multirow{5}{*}{ Ext. Distal } & Coxal & 1 & 1 & 1 & 1 & 1 & 1 & 2 & 1 & 1 \\
& Fémur & 1 & 1 & 1 & 2 & 2 & 1 & 1 & 1 & 1 \\
& Radioulna & - & - & - & 1 & 1 & 1 & - & - & - \\
& Tibia & - & - & - & 2 & 2 & 1 & - & - & - \\
& Carpos & - & - & - & 2 & 2 & 1 & 2 & 2 & 1 \\
& Tarsos & - & - & - & 1 & 1 & 1 & - & - & - \\
& Metapodios & - & - & - & 2 & 2 & 1 & - & - & - \\
& Falange 1 & - & - & - & 2 & 2 & 1 & 1 & 1 & 1 \\
& Falange 2 & - & - & - & 1 & 1 & 1 & - & - & - \\
& Falange 3 & - & - & - & 1 & 1 & 1 & - & - & - \\
& Falange Indet. & - & - & 1 & 1 & 1 & & - & - & - \\
& Total & 4 & 4 & 1 & 35 & 27 & 3 & 18 & 16 & 5 \\
\hline
\end{tabular}

incluyendo Ma. patachonica y Camelidae indet. En esta discusión se deja explícitamente fuera a My. darwini del que sólo se distinguen huesecillos dérmicos, especimenes que aún cuando poseen evidente deterioro digestivo, podrían encontrarse relacionados con el aprovechamiento del cuero que hacen algunos carnívoros del género Dusicyon y Puma (Borrero 1990; Martin 1998), por lo que no es posible asegurar un acceso a unidades anatómicas de este Mylodontinae.

En este punto es importante detenerse en la frecuencia de partes anatómicas y la representación de las distintas presas en la muestra. Como se aprecia en la Tabla 4, el 57,47\% de los elementos identificados corresponden a taxones del Grupo 2 (entre 70-140 Kg), es decir guanaco/huemul. El Grupo 3, se encuentra representado a través de 16 elementos (34\%) los que dan cuenta de cinco individuos. Dos de éstos corresponden a ejemplares juveniles de Hippidion saldiasi y uno de Camelidae indet.

La Fig. 6 grafica la frecuencia de partes esqueletaria de acuerdo a los segmentos mencionados en la metodología de los grupos 2 y 3 , comparado con datos de madrigueras actuales de puma (Borrero y Martin 1997), Pa. onca? del Pleistoceno de
Magallanes (Borrero et al. 1997) y Homotherium serum del Pleistoceno de Texas (Marean y Ehrhardt 1995). El Grupo 2 se encuentra representado por todos los segmentos de la carcasa (Fig. 6a), encontrándose mayormente registrados las porciones de las extremidades. En contraste, el Grupo 3, se encuentra dominado casi exclusivamente por el segmento craneal (Fig. 6b). Esta diferencia apuntaría, a nuestro juicio, a un acceso diferencial de la carcasa, en donde los individuos con un peso menor a 150 $\mathrm{Kg}$, son trasladados completos a las madrigueras, mientras que aquellos de mayor peso son transportados selectivamente, ingresando sólo unidades de menor rendimiento cárneo pero ricas en grasa. La comparación con las frecuencias de referencia permite establecer paralelos entre el Grupo 2 y las madrigueras de Pu. Concolor y Pa. Onca (Fig. 6c y d), y el Grupo 3 con aquella de Homotherium (Fig. 6e). Más que la presencia de dos taxones independientes, creemos que se trata de un solo carnívoro que utiliza distintas estrategias de caza/transporte de taxones. Hemos discutido que tanto Pu. concolor como Pa. onca depredan principalmente sobre artiodáctilos de tamaño medio, presas que pueden manejar y eventualmente trasladar hasta lugares 

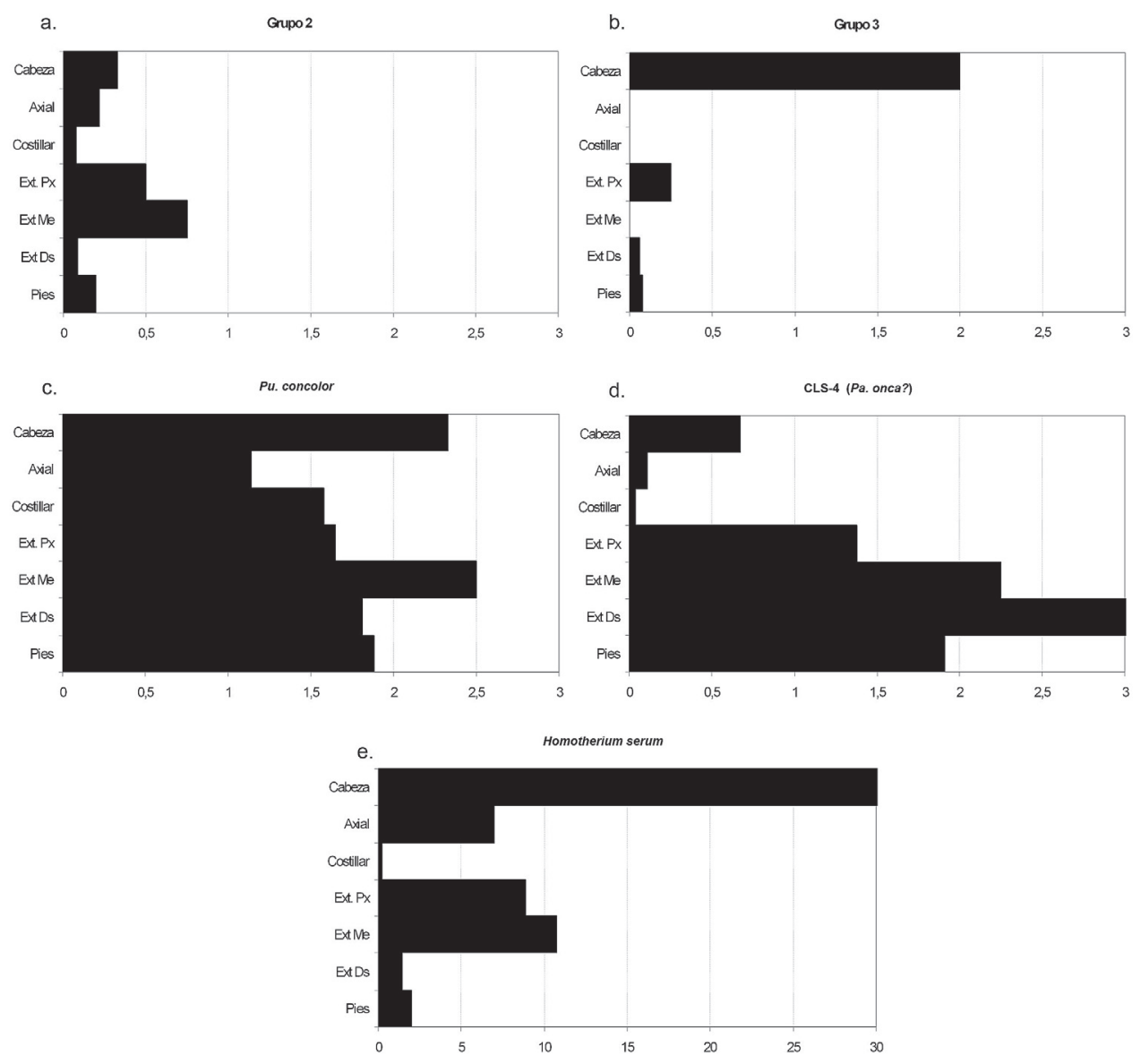

Fig. 6. Frecuencia de segmentos analíticos (MNE observado/MNE esperado) de los Grupos 2 y 3 , y conjuntos actualísticos y fósiles (ver el texto para explicación).

más aptos para su consumo. Difícilmente podrán cazar y desplazar taxones de cerca de una tonelada de masa, pero sí podrían acceder a ellos de manera secundaría, es decir carroñeando carcasas. De esta manera, trasladarían hacia las madrigueras segmentos anatómicos de menor volumen y por tanto más manejables. Stiner (1991) ha argumentado que una presencia mayoritaria de fragmentos craneales y mandibulares en madrigueras es reflejo de actividades de carroñeo, ya que éstas normalmente quedan en el lugar de matanza, situación que se acomoda muy bien a lo observado en cueva Las Guanacas. No es posible descartar a priori la presencia de un depredador mayor, como Smilodon, aunque la supuesta especialización de estos taxones hacia presas más grandes difiere de lo observado aquí. Aún cuando Smilodon depredara por igual taxones de distintos tamaños, sería esperable encontrar una frecuencia de partes similar para todas las especies, independiente de su masa corporal.

Como último elemento para la discusión, nos detendremos en los especimenes que presentan huellas de ácidos digestivos. Hasta la fecha, la totalidad de los trabajos tafonómicos realizados con fecas de carnívoros han intentado caracterizar sus conjuntos escatológicos utilizando la frecuencia de partes esqueletarias, la fragmentación y/o la incidencia de ácidos digestivos (Andrews 1990; Montalvo et al. 2007; Schmitt y Juell 1994). Éste último atributo solamente ha sido cuantificado en términos de su representación en relación al total de unidades y su intensidad. No obstante, el tamaño de las piezas que presentan huellas de ácido (fundamentalmente pitting), puede ser igualmente utilizado como indicador para 


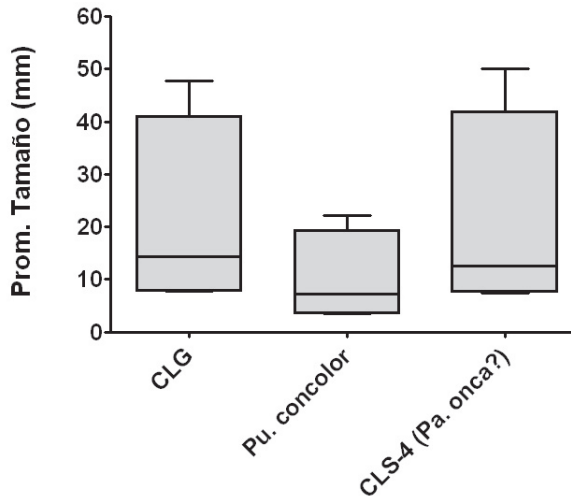

Fig. 7. Estadísticas descriptivas (media, máximo, mínimo y desviación) del promedio del largo y ancho de los especimenes con huellas de ácidos digestivos de cueva Las Guanacas (NISP:35) comparados con datos actualísticos de Pu. concolor (NISP:189) y Lago Sofía 4 (NISP:179) (Labarca et al. en prep.).

discernir entre distintos depredadores, asumiendo que existe una relación entre el porte del animal, sus fecas y el contenido de éstas (Labarca et al. en prep.). De esta manera, se obtuvo para cada uno de los especimenes con huellas de pitting por ácidos digestivos de cueva Las Guanacas, el promedio entre su largo y ancho máximo siguiendo la propuesta de Labarca et al. (en prep.), los que fueron comparados con datos actualísticos de Puma concolor y con información similar obtenida del yacimiento paleontológico de Lago Sofía 4 (Labarca et al. en prep.), en donde el análisis tafonómico indicaría la presencia de Panthera onca (Borrero et al. 1997) (Fig 7). Se observa en la Fig. 7 que los resultados de las estadísticas descriptivas (media, máximo, mínimo y desviación estándar) de cueva Las Guanacas se alejan de aquellas obtenidas para los conjuntos escatológicos actualísticos de puma, acercándose, en cambio, a las registradas en cueva Lago Sofía 4.

\section{CONCLUSIÓN}

Se documentan en las capas finipleistocénicas de cueva Las Guanacas tanto taxones extintos como vivientes. Entre los primeros se incluyen Hippidion saldiasi, My. darwini, Ma. patachonica y Camelidae indet. Entre los segundos se identificaron D. culpaeus, P. pudu, Hippocamelus bisulcus y L. guanicoe. El análisis de las modificaciones observables en la superficie de los restos óseos descartó una depositación cultural de éstos, siendo el principal agente responsa- ble de este proceso uno o varios carnívoros. En este sentido, la frecuencia de partes, taxones presentes, tipo de modificaciones e incidencia en la muestra permiten sugerir tentativamente que el principal depredador responsable de la acumulación de los restos habría sido un félido de tamaño medio, posiblemente Panthera onca, taxón que habría implementado una doble estrategia de adquisición de su alimento: caza de artiodáctilos medianos y posiblemente carroñeo de taxones mayores. Aparentemente otro carnívoro de menor tamaño habría participado en la conformación del registro y/o habría carroñeado los restos dejados por el depredador mayor e incluso ocupado la cueva como un frugal dormidero, como puede ser el caso de D. culpaeus.

La información palinológica indica que el retiro de los hielos glaciares habría comenzado a partir de los 18.000 cal AP. (Markgraf et al. 2007). Los fechados radiocarbónicos taxón provenientes del cercano yacimiento de Baño Nuevo 1 y los presentados aquí demuestran que el sector se encontraba habitado por mamíferos por lo menos desde los $13.500 \mathrm{AP}$ (Mena y Stafford 2006). Pese a que se trata de un rango temporal considerable, en términos generales la información que disponemos sobre el Pleistoceno final aisenino es escasa y fragmentaria. Este trabajo constituye un ejercicio teórico-metodológico orientado a comenzar a llenar este vacío, por lo menos en lo que respecta a las relaciones tróficas de una parte del elenco faunístico finipleistocénico. En este sentido, más que conclusiones fehacientes respecto de los agentes que generaron el registro paleontológico de Las Guanacas hacia el 13.200 AP, creemos que los lineamientos esbozados aquí deben ser el punto de partida para la generación de hipótesis de trabajo que guíen futuras investigaciones tanto en este como en nuevos yacimientos aun por descubrir.

\section{AGRADECIMIENTOS}

Agradecemos a Lucas Pomi y Marcelo Reguero (UNLP), por permitirnos el estudio de los materiales paleontológicos depositados en el Museo de La Plata (Argentina). María Teresa Alberdi (CSIC) facilitó las medidas de molares del género Hippidion. Kemel Sade, colaboró con el mapa de la Figura 1. Mariana Mondini y Mary Stiner proporcionaron bibliografía valiosa para la realización de este trabajo. Luis Borrero revisó una primera versión de éste. 
APÉNDICE 1. Medidas (mm) de los especímenes paleofaunísticos identificados.

TABLA 1. Medidas (mm) de molares de H. saldiasi de cueva Las Guanacas. Ls: longitud mesio-distal en superficie; As: anchura buco-lingual en superficie; Lb: longitud mesio-distal en la base; Ab: anchura buco-lingual en la base; $\mathrm{H}$ : altura del diente; LPr: longitud mesio-distal del protocono en superficie; Pli: pliegue caballuno; PLs: número de pliegues de las fosetas;

Apre: prefoseta anterior; Dpre: prefoseta distal; Apost: postfoseta anterior; Dpost: postfoseta distal; Hy: hypocono.

\begin{tabular}{|c|c|c|c|c|c|c|c|c|c|c|c|c|c|}
\hline \multirow{2}{*}{ Espécimen } & \multirow{2}{*}{ Sigla } & \multirow{2}{*}{ Ls } & \multirow{2}{*}{ As } & \multirow{2}{*}{$L b$} & \multirow{2}{*}{$A b$} & \multirow{2}{*}{$H$} & \multirow{2}{*}{$L P r$} & \multirow{2}{*}{$P l c$} & \multicolumn{4}{|c|}{ PLs Fosetas } & \multirow{2}{*}{ Hy } \\
\hline & & & & & & & & & Apre & Dpre & Apost & Dpost & \\
\hline M2i & 12B-N16-C10 & 24,7 & 25,6 & 24,7 & 26,2 & 40,3 & 9,8 & 0 & 0 & 0 & 0 & 0 & Oval estrang \\
\hline P2d & 12B-N15-C10 & 32,2 & 22,3 & 31 & 21,9 & 50,4 & - & 0 & 0 & 0 & 0 & 0 & Redond. \\
\hline
\end{tabular}

TABLA 2. Medidas ( $\mathrm{mm}$ ) de distintos especimenes de Camelidae indet. de cueva Las Guanacas. Bp: Ancho proximal; BFp: Ancho proximal de la carilla articular; Dp: Profundidad proximal; DC: Profundidad del caput femoral; GD: Profundidad máxima; GB: Ancho máximo; GH: Altura máxima.

\begin{tabular}{|c|c|c|c|c|c|}
\hline \multirow{2}{*}{$\begin{array}{ll} & \text { Espécimen } \\
\mathrm{m} 2 \mathrm{i} & \end{array}$} & \multicolumn{4}{|c|}{ Medidas } & \multirow{2}{*}{ Observaciones } \\
\hline & Ls & As & $\mathbf{L b}$ & $\mathbf{A b}$ & \\
\hline Sin ref. & 27,6 & 14,4 & 21,3 & 20,4 & Desgaste leve \\
\hline Fémur & DC & & & & \\
\hline \multirow[t]{2}{*}{ 11A-N11-C9 } & 43,3 & & & & Sin fusionar \\
\hline & GD & GB & GH & & \\
\hline Segundo carpiano & 21,37 & 16,54 & 8,52 & & \\
\hline \multicolumn{6}{|l|}{ Cuarto Carpiano } \\
\hline 11B-N16-C10 & 41,3 & 29 & 91,4 & & c/ huellas de ácido \\
\hline
\end{tabular}

TABLA 3. Medidas (mm) de la primera Falange de Macrauchenia patachonica de cueva Las Guanacas.

\begin{tabular}{lccc}
\hline \multicolumn{1}{c}{ Espécimen } & Medidas \\
\hline Falange 1 & Bp & BFp & Dp \\
10A'-N16-C10 & 35,4 & 34,5 & 36,4 \\
\hline
\end{tabular}

TABLA 4. Medidas (mm) de distintos especimenes de Pudu pudu de cueva Las Guanacas. Abreviaturas como en Tabla 1 y 2. Bp: Ancho máximo proximal.

\begin{tabular}{|c|c|c|c|c|c|}
\hline \multirow{2}{*}{$\begin{array}{ll} & \text { Espécimen } \\
\mathrm{m} 3 \mathrm{~d} & \end{array}$} & \multicolumn{4}{|c|}{ Medidas } & \multirow[t]{2}{*}{ Observaciones } \\
\hline & Ls & As & Lb & $\mathbf{A b}$ & \\
\hline 10A-N18-C10 & 13,1 & 6,5 & - & - & Muy desgastado \\
\hline $\mathrm{m} 3 \mathrm{i}$ & 13,6 & 6,5 & & & Muy desgastado \\
\hline 10A-N19-C10 & & & & & \\
\hline Fémur & DC & $\mathbf{B p}$ & & & \\
\hline 10A-N17-C10 & 9,7 & 20,3 & & & Semifusionado \\
\hline
\end{tabular}


TABLA 5. Medidas (mm) del esqueleto postcraneal de D. culpaeus de cueva Las Guanacas. GL: Largo máximo, Bp: Ancho máximo proximal; Dp: Profundidad máxima distal; Bd: Ancho máximo distal; Dd:

Profundidad máxima distal; Bt: Ancho máximo de la tróclea; GB: ancho máximo, GLP: Largo máximo del proceso glenoideo; BG: Ancho máximo de la cavidad glenoidea; HS: altura a través de la espina.

\begin{tabular}{|c|c|c|c|c|c|c|c|c|c|c|c|}
\hline Espécimen & & & & & & Medida: & & & & & \\
\hline & GL & Bp & Dp & Bd & Dd & Bt & Ll & GB & GLP & BG & HS \\
\hline Escápula & & & & & & & & & & & \\
\hline 11A-N24-C11 & & & & & & & & & 20,7 & 11,9 & 91,5 \\
\hline 11B-N16-C10 & & & & & & & & & & 11,8 & \\
\hline 11A-N25-C11 & & & & & & & & & 20,8 & 11,7 & \\
\hline Húmero & & & & & & & & & & & \\
\hline 11B-N16-C10 & 122,9 & 25,2 & 26,5 & 24,4 & 17,8 & 19 & & & & & \\
\hline 11A-N25-C12 & 131,6 & & 26,3 & 24,9 & 18,5 & 18,1 & & & & & \\
\hline Tibia & & & & & & & & & & & \\
\hline 11A-N24-C11 & 145,7 & 25,2 & 26,5 & 17,5 & 12,1 & & 139,7 & & & & \\
\hline Calcáneo & & & & & & & & & & & \\
\hline 11A-N25-C12 & 33,74 & & & & & & & 12,69 & & & \\
\hline Astrágalo & & & & & & & & & & & \\
\hline 11A-N26-C12 & 21 & 11,6 & & & & & & & & & \\
\hline
\end{tabular}




\section{BIBLIOGRAFÍA}

ALBERDI, M.T. y A. PRIETO. 2000. Restos de Hippidion saldiasi en las cuevas de la Patagonia chilena. Anales del Instituto de la Patagonia, Serie Ciencias Humanas 28:147-171.

ALBERDI, M.T. y J.L PRADO 2004. Los caballos fósiles de América del Sur. Una historia de 3 millones de años. Universidad Nacional del Centro de la Provincia de Buenos Aires, INQUAPA Serie monográfica 3.

ALTAMIRANO, A. 1983. Guía Osteológica de Cérvidos Andinos. Universidad Nacional Mayor San Marcos. Lima.

ANDREWS, P. 1990. Owls, Caves and Fossils. Predation, Preservation y Accumulation of Small Mammals Bones in Caves. British Museum of Natural History, United Kingdom.

BATE, L.F. 1970. Primeras Investigaciones sobre el Arte rupestre de la Patagonia Chilena. Anales del Instituto de la Patagonia 1: 15-25

BEHRENSMEYER, A. K. 1978. Taphonomic and Ecologic Information from Bone Weathering. Paleobiology 4(2):150-162.

BEHRENSMEYER, A. K., K. D. GORDON y G. T. YANAGI 1986. Trampling as a cause of bone surface damage and pseudotools. Nature 319:768-771.

BENAVENTE, M. A., L. ADARO, P. GECELE y C. CUNAZZA 1993. Contribución a la determinación de especies animales en arqueología: Familia Camelidae y Taruca del Norte. Departamento Técnico de Investigación, Universidad de Chile, Santiago.

BERMAN, W.D. y E.P. TONNI 1987. Canis (Dusicyon) avus, Brumeister, 1864 (Carnívora, Canidae) en el Pleistoceno tardío y Holoceno de la Provincia de Buenos Aires. Aspectos sistemáticos y bioestratigráficos. Ameghiniana 24(3-4):245-250.

BERTA, A. 1987. The sabercat Smilodon gracilis and discussion of its relationship (Mammalia, Felidae, Smilodontini). Bulletin of the Florida state museum, Biological Science 31(1):1-63. Florida.

BINFORD, L. R. 1981. Bones: ancient men and modern myths. Academic Press, New York.

BIRD, J. 1993. Viajes y arqueología en chile austral. Ediciones de la Universidad de Magallanes. Punta Arenas.

BORRERO, L.A. 1990. Taphonomy of guanaco bones in Tierra del Fuego. Quaternary Research 34:361-371.

BORRERO, L.A., F. M. MARTIN y A. PRIETO 1997. La Cueva Lago Sofía 4, Última Esperanza: una madriguera de felino del Pleistoceno tardío. Anales del Instituto de la Patagonia, Serie Ciencias Humanas 25:103-122.
BORRERO, L.A, F. MARTín y J. VARGAS 2005. Tafonomía de la interacción entre pumas y guanacos en el Parque Nacional Torres del Paine, Chile. Magallania 33:95-114.

BRAIN, C. K. 1981. The Hunters or the Hunted? An Introduction to South African Cave Taphonomy. University of Chicago Press, Chicago.

CABRERA A. 1932. Sobre los camélidos fósiles y actuales de la América Austral. Revista del Museo de la Plata 33:89-117.

CARTAJENA, M.I. 2002. Los conjuntos arqueofaunísticos del Arcaico Temprano en la Puna de Atacama, Norte de Chile. Tesis Doctoral inédita. Área de Historia y Cultura. Universidad de Berlín, Berlín.

CARTELLE, C y G.A BOHÓRQUEZ 1986. Presença de ossículos dérmicos em Eremotherium laurillardi (Lund) Cartelle \& Bohórquez 1982 (Edentanta, Megatheriidae). Iheringía, serie Geológica 11:9-14.

CARTELLE, C. y G. DE IULIIS 2006. Eremotherium laurillardi (Lund) (Xenarthra, Megatheriidae), the panamerican giant ground sloth: taxonomic aspects of the ontogeny of skull and dentition. Journal of Systematic Palaeontology 4:199-209.

CASCELLI de ACEVEDO, F. y D.L. MURRAY 2007. Spatial organization and food habitats of jaguars (Panthera onca) in a floodplain forest. Biological conservation 137:391-402.

CAVIGLIA, S. 1985-86. Nuevos restos de cánidos tempranos en sitios arqueológicos de Fuego- Patagonia. Anales del Instituto de la Patagonia, Serie Ciencias Sociales 16: 85-93.

CLEGHORN, N. y MAREAN, C. W. 2007. The destruction of human-discarded bone by carnivores: The growth of a general model for bone survival and destruction in zooarchaeological assemblages. En: African taphonomy: A tribute to the career of C. K. "Bob" Brain, editado por T. R. Pickering, N. Toth y K. Schick, pp. 13-42. Bloomington: Stone Age Press.

EIROA, J., J. BACHILLER, I. CASTRO y J. LOMBA 1999. Nociones de tecnología prehistórica. Ariel Historia. España.

ESTEBAN, G. 1996. Revisión de los Mylodontinae cuaternarios (Edentata, Tardigrada) de Argentina, Bolivia y Uruguay. Sistemática, Filogenia, Paleobiología, Paleozoogeografía y Paleoecología. Tesis Doctoral inédita. Facultad de Ciencias Naturales. Instituto Miguel Lillo, Tucumán.

FARIÑA R.A., S.F. VIZCAÍNO y M.S. BARGO 1998. Body mass estimations in Lujanian (Late Pleistocene-Early Holocene of South America) mammal megafauna. Mastozoología Neotropical 5: 87-108. 
FUENTES, F. y F. MENA 2008. Movilidad en cazadores-recolectores: el caso de cueva las Guanacas (XI Región Aisén, Chile). Trabajo presentado a las I Jornadas Nacionales de Arqueología de Cazadores-Recolectores. Santiago.

GARLA, R.C., E.Z. SETZ y N. GOBBI 2001. Jaguar (Panthera onca) food habits in Atlantic Rain forest of Southeastern Brazil. Biotropica 33(4):691-696.

GONZÁLEZ, G., J.C. TORRES-MURRA y A. MUÑOZ-PEDREROS 2000. Orden Artiodactyla. En Mamíferos de Chile ,editado por A. Muñoz-Pedreros y J. Yánez, pp. 189-206. CEA Ediciones.

GRAHAM, R.W. y E.L. LUNDELIUS 1984. Coevolutionary disequilibrium and Pleistocene extinctions. En Quaternary extinctions: A prehistoric revolution, editado por P.S. Martin y R.G. Klein, pp. 354-404). University of Arizona Press. Tucson, Arizona,

GUÉRIN, C. y M. FAURE 1999. Palaeolama (Hemiauchenia) niedae nov. sp., nouveau camelidae du nordeste brésilien et sa place parmi les lamini d'Amérique du Sud. Geobios 32 (4): 620-659.

HAYNES, G. 1983. A guide for differentiating mammalian carnivore taxa responsible for gnaw damage to herbivore limb bones. Paleobiology 9:164-172.

IRIARTE, J.A., W.E. JOHNSON y W.L. FRANKLIN 1991. Feeding ecology of the Patagonia puma in southermost Chile. Revista Chilena de Historia Natural 64: 145-156.

KAUFMANN, C. 2004. La fusión ósea como indicador de edad y estacionalidad en guanaco (Lama guanicoe). En Actas de las V Jornadas de Arqueología de la Patagonia, editado por M. T. Civalero, P. Fernández y G. Guráieb, pp. 477-488. Instituto Nacional de Antropología y Pensamiento Latinoamericano, Buenos Aires.

LABARCA, R. 2008. Los mamíferos del Plio-Pleistoceno de Chile. En Los vertebrados fósiles de Chile, editado por D. Rubilar y M. Salaberry. En prensa.

LABARCA, R., A. PRIETO y R. VILLALOBOS Tafonomía de fecas de Puma concolor y su aplicación en contextos arqueológicos y paleontológicos. En preparación

LEVINE, M.A. 1982. The use of crown height measurements and eruption-wear sequences to age horse teeth. En Ageing and Sexing Animal Bones from Archaeological Sites, editado por B.Wilson, C. Grigson y S. Payne, pp. 223-250. BAR British Series 109. Oxford: British Archaeological Reports.

LÓPEZ, P. 2008. Tafonomía de los mamíferos extintos del sitio Baño Nuevo 1 (XI Región-Chile). Trabajo presentado al I Taller de zooarqueología en Chile. Santiago.

LYMAN, R. L. 1994. Vertebrate taphonomy. Cambridge University Press, Cambridge.
MAREAN, C.W. y C.L. EHRHARDT 1995. Paleoanthropological and paleoecological implications of the taphonomy of a sabertooth's den. Journal of Human Evolution 29:515-547.

MARKGRAF, V., C. WITHLOCK y S. HABERLE 2007. Vegetation and fire history during the last 18,000 cal yr B.P in southern Patagonia: Mallín Polloux, Coyhaique, Province Aisén (4541'30" S, 7150'30"' W, 640 m elevation). Palaeogeography, Palaeoclimatology, Palaeoecology 254:492-507.

MARTIN, F. 1998. Madrigueras, dormideros y letrinas: Aproximación a la tafonomía de zorros. En Arqueología de la Patagonia Meridional (Proyecto "Magallania"), editado por L.A. Borrero, pp. 73-96. Ediciones Búsqueda de Ayllu.

MARTIN, F. y L.A. BORRERO 1997. A puma in southern Patagonia: implications for the archaeological record. Current Anthropology 38:453-461.

MARTIN, F., A. PRIETO, M. SAN ROMÁN, F. MORELLO, F. PREVOSTI, P. CÁRDENAS y L.A. BORRERO. 2004. Late-Pleistocene Megafauna at Cueva del Puma, Pali-Aike Lava Field, Chile. Current Research in the Pleistocene 21:101-103.

MASSONE, M. 2004. Los Cazadores después del Hielo. Colección de Antropología 7, Centro de Investigación Barros Arana. Ediciones de la DIBAM.

MENA, F. 1983. Excavaciones arqueológicas en Cueva Las Guanacas (RI-16) XI Región. Anales del Instituto de la Patagonia 14:65-75.

MENA, F. 1992. Mandíbulas y maxilares: un primer acercamiento a los conjuntos arqueofaunísticos del Alero Fontana. Boletín del Museo Nacional de Historia Natural 43:179-191.

MENA, F. 1997. Paleoindio en Aisén: Una reevaluación. Trabajo presentado al XIV Congreso Nacional de Arqueología de Chile. Copiapó.

MENA, F. y T. STAFFORD 2006. Contexto estratigráfico y fechación directa de esqueletos humanos del Holoceno Temprano en Cueva Baño Nuevo (Patagonia Central, Chile) En Segundo simposio Internacional del Hombre Temprano en América, pp. 139-154. CONACULTAINAH, México.

MENEGAZ, A.N. 2000. Los camélidos y cérvidos del cuaternario del sector bonaerense de la Región Pampeana. Tesis Doctoral inédita. Facultad de Ciencias Naturales y Museo de La Plata, La Plata.

MENGONI, G. 1999. Cazadores de Guanacos de la estepa Patagónica. Colección tesis doctorales, Sociedad Argentina de Antropología. 
MENGONI, G., M.J. FIGUERERO. M.V. FERNÁNDEZ y P. CHÁVEZ. 2008. Carácter de las ocupaciones en el área de Los Antiguos-Monte Zeballos y Paso Roballos (Prov. Santa Cruz, Argentina). Trabajo presentado a las VII Jornadas de Arqueología de la Patagonia, Ushuaia.

MONDINI, M. 1995. Artiodactyl prey transport by foxes in Puna rock shelters. Current Anthropology 36 (3):520-524.

MONDINI, M. 2003. Modificaciones óseas por carnívoros en la Puna argentina, Una mirada desde el presente a la formación del registro arqueofaunístico. Mundo de Antes 3:87-108.

MONTALVO, C., M. PESSINO y V. GONZÁLEZ 2007. Taphonomic analysis of remains of mammals eaten by pumas (Puma concolor Carnivora, Felidae) in central Argentina. Journal of Archaeological Science 34:2151-2160.

NARANJO, J.A. y C. STERN 1998. Holocene explosive activity of Hudson volcano, southern Andes. Bulletin of Volcanology 59: 291-306

PACHECO TORRES, V., A. ALTAMIRANO ENCISO y E. GUERRA PORRAS 1986. The osteology of South American camelids. Institute of Archaeology University of California, Los Angeles.

PALMEIRA, F., P. CRAWSHAW, C. HADDAD, K.M. FERRAZ y L. VERDADE 2008. Cattle depredation by puma (Puma concolor) and jaguar (Panthera onca) in central-western Brazil. Biological conservation 141: 118-125.

PEYTON, B. 1980. Ecology, distribution, and food habits of spectacled bears Tremarctos ornatus, in Peru. Journal of Mammology 61:639-652.

PREVOSTI, F. y S. VIZCANÍO 2006. Paleoecology the large carnivore guild from the late Pleistocene of Argentina. Acta Paleontologca Polonica 51:407-422.

PREVOSTI, F., SOIBELZON, L., PRIETO, A., SAN ROMÁN, M. \& MORELLO, F. 2003. The Southermost Bear: Pararctotherium (Carnivora, Ursidae, Tremarctinae) in the Latest Pleistocene of Southern Patagonia, Chile. Journal of Vertebrate Paleontology 23(3): 709-712.

PURDUE, J.R. 1983. Epiphyseal Closure in White-Tailed Deer. The Journal of Wildlife Management 47(4): 1207-1213.

QUINTANA, V., J. YÁNEZ y M. VALDEVENITO 2000. Orden carnívora. En Mamíferos de Chile, editado por A. MuñozPedreros y J. Yánez, pp. 155-188. CEA Ediciones.
RADLOFF, F.G y J.T. DU TOIT 2004. Large predators and their prey in a southern African savanna: a predator's size determines its prey size range. Journal of Animal Ecology 73:410-423

REYES, O., C. MÉNDEZ, H. VELÁSQUEZ y V. TREJO 2007. El Chueco 1: un asentamiento multicomponente en la estepa occidental de Patagonia Central (11.400 a 2.700 años cal. ap, 44으). Magallania 35 (1): 107-119.

RINDERKNECHT, A. 2000. La presencia de osteodermos en las extremidades posteriores de Glyptodon clavipes Owen, 1839 (Mammalia: Cingulata). Ameghiniana 37(3): 369-373.

SADE, K. 2008. Cazadores Extintos de Aysén Continental: propuesta de poblamiento Ediciones Nire Negro, Coyhaique.

SCHERER, C.S., FERIGOLO, J., RIBEIRO, A.M. y CARTELLE, C. 2007. Contribution to the knowledge of Hemiauchenia paradoxa (Artiodactyla, Camelidae) from the Pleistocene of southern Brazil. Revista brasileira de paleontología 10(1):35-52.

SCHMITT, D.N. y K.E. JUELL 1994. Toward the identification of coyote scatological faunal accumulations in archaeological contexts. Journal of Archaeological Science 21:249-262.

SCOGNAMILLO, D., I.E. MAXIT., M. SUNQUIST y J. POLISAR 2001. Coexistence of jaguar (Panthera onca) and puma (Puma concolor) in a mosaic landscape in the Venezuela llanos. Journal of the Zoological Society of London 259: 269-279.

STINER, M. 1991. Food procurement and transport by human and non-human predators. Journal of Archaoelogical Science 18:455-482.

TREJO, V. y D. JACKSON 1998. Cánidos patagónicos: identificación taxonómica de mandíbulas y molares del sitio arqueológico Cueva Baño Nuevo-1 (Alto Nirehuao, XI Región). Anales del Instituto de la Patagonia, Serie Ciencias Humanas 26:181-194.

TURNER, A. y M. ANTÓN. 1997. The big cats and their fossil relatives. Columbia University Press, New York.

Von den DRIESCH, A. 1999. A guide to the measurement of animal bones from archaeological sites. Peabody Museum Bulletin 1. Peabody Museum of Archaeology and Ethnology Harvard University. 137 pp. Segunda edición. 